\title{
A DISCUSSION ON THE PHONON DENSITY OF STATES OF AMORPHOUS GERMANIUM FOR THE INFRARED RANGE
}

\author{
M. A. GRADO-CAFFARO* and M. GRADO-CAFFARO \\ Scientific Consultants, C/Zulio Palacios, 11, 9으 B 28029-MADRID (SPAIN)
}

(Received 18 November 1997; In final form 23 January 1998)

A theoretical formulation for the phonon density of states of amorphous germanium in the infrared range is proposed. This formulation is based upon the quasi-harmonic approximation and is compared with previous results.

Keywords: Phonon density of states; amorphous germanium; infrared range; quasiharmonic approximation

\section{INTRODUCTION}

It is well-known that the phonon (or vibrational) density of states of amorphous tetrahedrally bonded semiconductors represents a crucial element to study optical absorption corresponding to structural disorder. In particular, $a$-germanium offers some interesting features from the point of view of optical absorption in the infrared range so that there is no dynamical disorder contribution to this absorption. By taking into account a well-known formula for the phonon density of states in the quasi-harmonic approximation, it is feasible to obtain an expression for the coefficient of optical absorption in $a$-Ge for the infrared region by considering only structural disorder contribution; this is the aim of this paper.

${ }^{*}$ Corresponding author. 


\section{THEORY}

By using atomic units and absorbing refractive index into the coefficient of optical absorption, we can write $\alpha=\omega \varepsilon_{2}$ where $\alpha(\omega)$ stands for absorption coefficient and $\varepsilon_{2}(\omega)$ denotes first-order infrared spectrum. In addition, by taking into account that for $a$-Ge this spectrum is entirely due to structural disorder [1, 2], we have [1, 3-5]:

$$
\alpha(\omega)=\frac{|\mu(\omega)|^{2}}{\omega} \cdot g(\omega)
$$

where $\mu(\omega)$ is the frequency-dependent dipole moment matrix element and $g(\omega)$ is the phonon density of states.

On the other hand, we can write $[4,6]$ :

$$
g(\omega)=\frac{1}{N} \sum_{n}^{N}\left|a_{n}\right|^{2} \delta\left(\omega-\omega_{n}\right)
$$

where $\omega_{n}$ is the vibrational eigenfrequency of the $n$th normal mode of the solid and $\delta$ stands for Dirac delta function. Eq. (2) refers to a quasi-harmonic approach. Moreover, matrix elements $a_{n}$ behave as dependent upon experiment and are also model-dependent [2].

Now by substituting formula (2) into formula (1), we get:

$$
\alpha(\omega)=\frac{|\mu(\omega)|^{2}}{N \omega} \cdot \sum_{n}^{N}\left|a_{n}\right|^{2} \delta\left(\omega-\omega_{n}\right) \quad(1 \leq n \leq N)
$$

when $\omega \rightarrow 0$, it is well-known that $|\mu(\omega)| / \omega$ tends to zero (see for example, Ref. [1]); then, from this fact is follows the evident condition $\lim _{\omega \rightarrow 0} \alpha(\omega)=0$ and that $(d \alpha / d \omega) \rightarrow 0$ as $\omega \rightarrow 0$ (by virtue of the L'Hôpital rule). Next we will examine the low margin of the farinfrared range; in this margin, it is well-known that $|\mu(\omega)|^{2}=k \omega^{2}$ where $k$ is a positive constant $[2,3,5]$ so that Eq. (3) becomes:

$$
\alpha(\omega) \approx k N^{-1} \omega \sum_{n}^{N}\left|a_{n}\right|^{2} \delta\left(\omega-\omega_{n}\right) .
$$

In addition, for the lowest frequencies in the far-infrared range there are many photons $(N \rightarrow \infty)$ so that, by Stolz's criterion, we have 


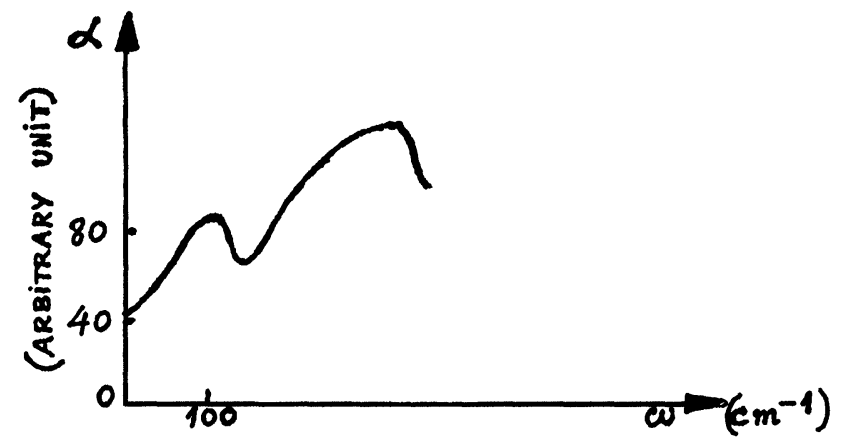

FIGURE 1 Optical absorption coefficient of $a$-Ge; this plot is experimental (after Ref. [6]).

$$
\begin{aligned}
\lim _{N \rightarrow \infty} \alpha & \approx k \omega \lim _{N \rightarrow \infty} \frac{\sum_{n=1}^{N}\left|a_{n}\right|^{2} \delta\left(\omega-\omega_{n}\right)-\sum_{n=1}^{N-1}\left|a_{n}\right|^{2} \delta\left(\omega-\omega_{n}\right)}{N-(N-1)} \\
& =k \omega \lim _{N \rightarrow \infty}\left[\left|a_{N}\right|^{2} \delta\left(\omega-\omega_{N}\right)\right]
\end{aligned}
$$

By employing numerical values corresponding to $a-\mathrm{Ge}$ and truncating delta function in formula (5), a straight line is obtained from this formula; our result is $\alpha(\omega) \approx 0.841 \omega\left(\omega\right.$ in $\left.\mathrm{cm}^{-1}\right)$ which agrees with Figure 1 (after Ref. [6]) for approximately $50 \mathrm{~cm}^{-1} \leq \omega \leq$ $100 \mathrm{~cm}^{-1}$.

\section{CONCLUSIONS}

Optical absorption in $a$-Ge for the far-infrared range has been discussed leading to an analytic expression for the coefficient of optical absorption corresponding to the low region of the above range. This expression agrees well with experiment between $50 \mathrm{~cm}^{-1}$ and $100 \mathrm{~cm}^{-1}$ approximately. However, below $50 \mathrm{~cm}^{-1}$ there is no agreement between our results and experiment after Ref. [6]. On the other hand, extrapolation of our method to other amorphous semiconductors is feasible so that it is possible to derive formulae which become appropriate to explain some features of these materials which may be regarded as unexplored aspects. 


\section{References}

[1] Mitra, S. S., Paul, D. K., Tsay, Y. F. and Bendow, B. (1974). AIP Conf. Proc., 20, 284-289.

[2] Grado-Caffaro, M. A. and Grado-Caffaro, M., Act. Pass. Electronic Comp., (in press).

[3] Grado-Caffaro, M. A. and Grado-Caffaro, M. (1992). Phys. Lett., A169, 399-401.

[4] Grado-Caffaro, M. A. and Grado-Caffaro, M. (1993). Mod. Phys. Lett., B7, 1203 1204.

[5] Grado-Caffaro, M. A. and Grado-Caffaro, M. (1994). Mod. Phys. Lett., B8, $247-$ 250.

[6] Stimets, R. W., Waldman, J., Lin, J., Chang, T. S., Temkin, R. J. and Connell, G. A. N. (1973). Solid State Commun., 13, 1485. 

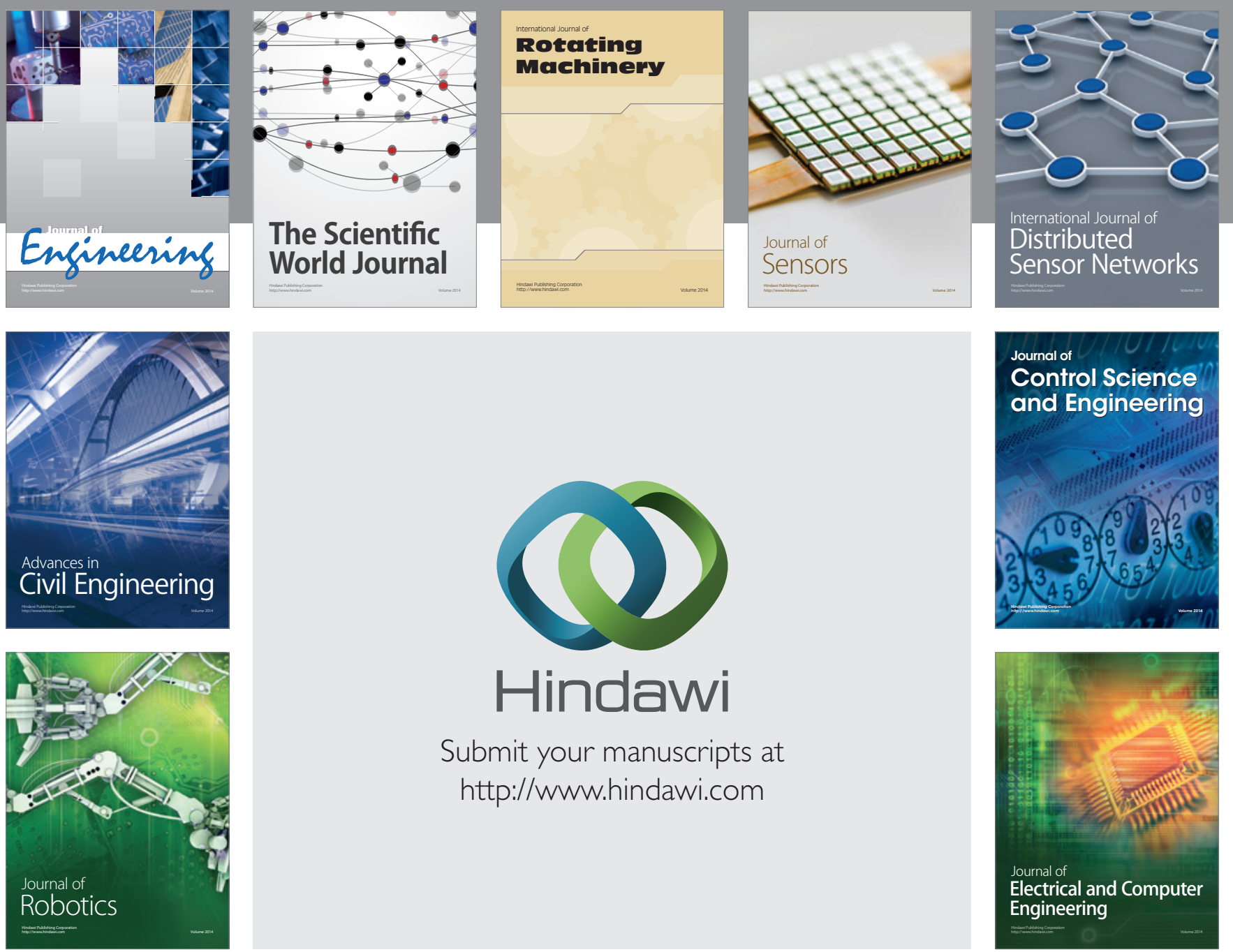

Submit your manuscripts at

http://www.hindawi.com
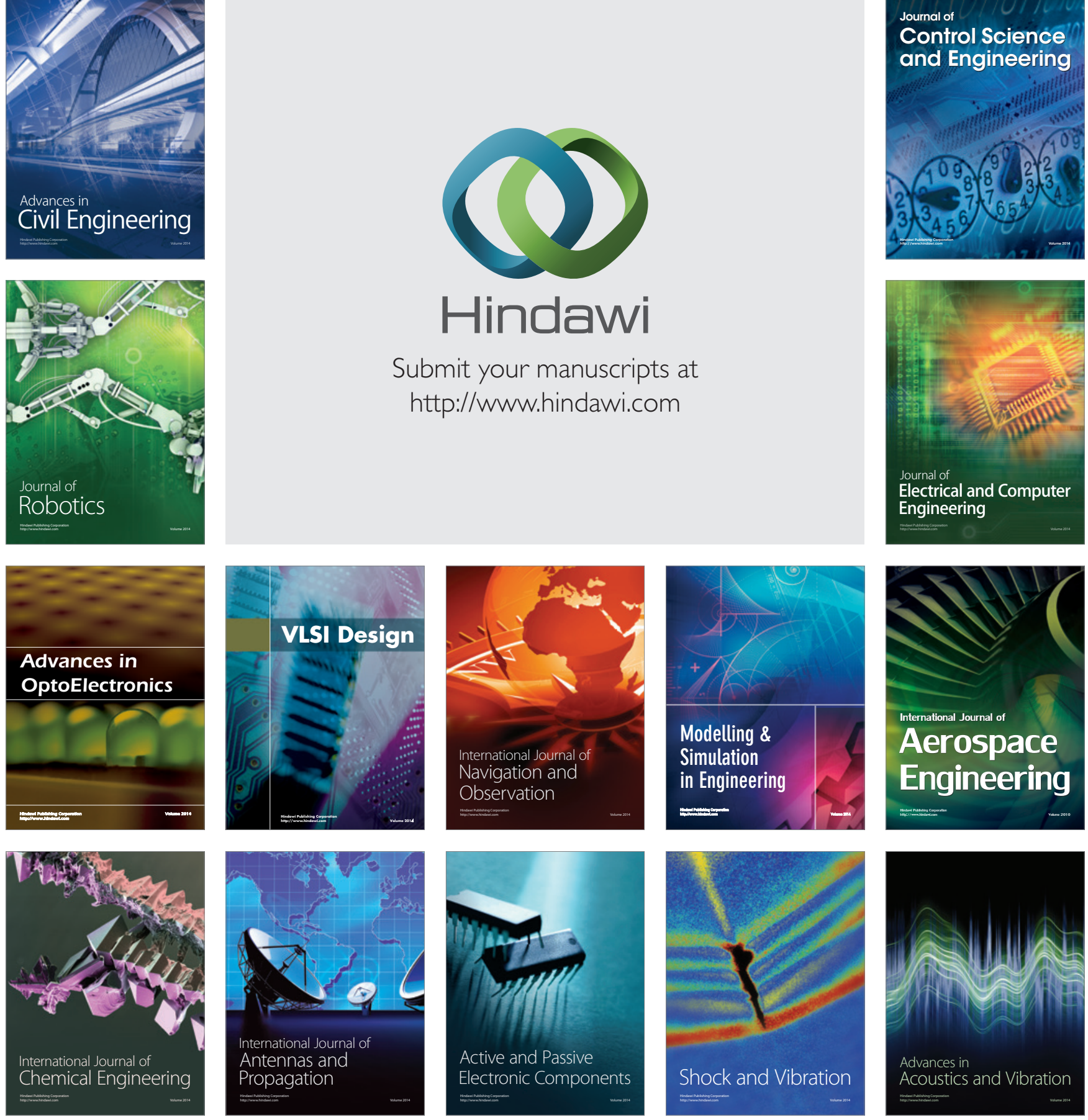